Proceedings of the Edinburgh Mathematical Society (2005) 48, 423-444 (C)

DOI:10.1017/S0013091504000513 Printed in the United Kingdom

\title{
A GENERAL FRAMEWORK FOR HOLOMORPHIC FUNCTIONAL CALCULI
}

\author{
MARKUS HAASE \\ Abteilung Angewandte Analysis, Universität Ulm, Helmholtzstraße 18, \\ 89069 Ulm, Germany (haase@mathematik.uni-ulm.de)
}

(Received 30 October 2004)

\begin{abstract}
We present an abstract approach to the construction of holomorphic functional calculi for unbounded operators and apply it to the special case of sectorial operators. In effect, we obtain a calculus for a much larger class of functions than was known before, including certain meromorphic functions. We discuss the role of topology. Then we prove in detail a composition rule $(f \circ g)(A)=f(g(A))$ which is the main result of the paper. This is done in such a way that the proof can easily be transferred to functional calculi for other classes of operators.
\end{abstract}

Keywords: functional calculus; sectorial operator; holomorphic semigroup

2000 Mathematics subject classification: Primary 47A60; 47D06

\section{Introduction}

The theory of functional calculus deals with the idea of 'inserting' an operator $A$ acting on a Banach space $X$ into certain complex-valued functions defined on a subset of $\mathbb{C}$. The reason why this idea is so appealing is that certain well-known and important constructions can be read in this way. Take, for example, a strongly continuous semigroup $(T(t))_{t \geqslant 0}$, which is frequently written in the form

$$
T(t)=\mathrm{e}^{t A} \quad(t \geqslant 0),
$$

where $A$ is the infinitesimal generator of the semigroup $T$. It seems as if $T(t)$ is obtained by somehow inserting the operator $A$ into the complex-valued function $\mathrm{e}^{t z}$. Other examples are the fractional powers $A^{\alpha}=\left(z^{\alpha}\right)(A)(\operatorname{Re} \alpha>0)$ or the logarithm $\log A=(\log z)(A)$ of a sectorial operator $A$ (see Definition 2.1).

Historically, the above examples emerged individually within the theory of evolution equations, with a separate definition for each of them. In special cases, such as self-adjoint operators on Hilbert spaces, they could be embedded into a general scheme of inserting operators into functions, comprising formulae like

$$
(f+g)(A)=f(A)+g(A) \quad \text { and } \quad(f g)(A)=f(A) g(A) .
$$


This general scheme, called functional calculus, has proved to be an invaluable tool. Dunford and Riesz had the idea to use the Cauchy-integral formula

$$
f(a)=\frac{1}{2 \pi \mathrm{i}} \int_{\Gamma} f(w) \frac{1}{w-a} \mathrm{~d} w
$$

and base on it the construction of a functional calculus in the Banach space setting by defining

$$
f(A):=\frac{1}{2 \pi \mathrm{i}} \int_{\Gamma} f(w) R(w, A) \mathrm{d} w
$$

(see [7, Section VII.3]). However, the operator $A$ under consideration still had to be bounded and the function $f$ had to be holomorphic on a neighbourhood of the spectrum $\sigma(A)$. Bade in $[3]$ overcame this restriction in the special case of 'strip type' operators (cf. also [10]). Summarized abstractly, $\Gamma$ is allowed to touch the spectrum of $A$ at certain 'singular' points (such as infinity in the case of an unbounded operator) and the decay of $f$ at those points is fast enough to compensate the growth of the resolvent there. Then an 'elementary' functional calculus is defined by (1.3). Afterwards a certain extension procedure is applied to include more general functions. McIntosh in [16] adapted this construction to the case of sectorial operators (see also [4]). The so-constructed calculus includes holomorphic semigroups, fractional powers and logarithms, and plays an important role in evolution equations and operator theory (see $[\mathbf{2}, \mathbf{6}, \mathbf{1 3}, \mathbf{1 4}]$ and the references therein).

The purpose of the present paper is twofold. First, we examine to some extent how (mainly holomorphic) functional calculi can be constructed, which results about them can be proved and which problems can arise. (We do not claim exhaustive treatment of these questions.) For illustration we use the model case of sectorial operators. The above-mentioned method for extending an 'elementary' functional calculus to a wider class of functions is given an axiomatic treatment (in $\S 3$ ). In effect, the basic rules of functional calculi (Theorem 3.6) appear as easy corollaries of the abstract results. The abstract approach allows one also to include even certain meromorphic functions, whence the functional calculi presented here are 'larger' than those which appear already in the literature. (This extension to meromorphic functions finally leads to a proof of a spectral mapping theorem in $[\mathbf{1 2}]$.)

Secondly, we formulate, prove and illustrate a composition rule

$$
(f \circ g)(A)=f(g(A))
$$

for our model functional calculus (Theorem 7.1). Together with the formulae (1.1) it paves the way for really calculating by means of the functional calculus (cf. Example 7.6). As far as we know, a proof for a general composition rule has not yet appeared in the literature (at least not in this generality). Let us also mention that the proof is generic so that similar proofs can be furnished for other functional calculi.

Let us fix some notation. For any open set $\Omega \subset \mathbb{C}$ we denote by $\mathcal{O}(\Omega)\left(\mathcal{M}(\Omega), H^{\infty}(\Omega)\right)$ the space of all holomorphic (meromorphic, bounded holomorphic) functions on $\Omega$. 
For $0<\omega \leqslant \pi$ let $S_{\omega}:=\{z \in \mathbb{C} \mid z \neq 0$ and $|\arg z|<\omega\}$ denote the open sector symmetric about the positive real axis with opening angle $\omega$. To also cover the case $\omega=0$ we define $S_{0}:=(0, \infty)$.

By 'operator' we always mean linear operator. For an operator $A$ on a Banach space $X$, we denote by $\mathcal{D}(A)$ its domain, by $\mathcal{N}(A)$ its kernel and by $\mathcal{R}(A)$ its range. We denote by $\mathcal{L}(X)$ the set of all bounded, fully defined operators on $X$. If $A \in \mathcal{L}(X)$, the number $r(A):=\sup \{|\lambda| \mid \lambda \in \sigma(A)\}$ is the spectral radius of $A$. Given operators $A, B$ on a Banach space we denote by $A+B$ its sum with the domain $\mathcal{D}(A+B)=\mathcal{D}(A) \cap \mathcal{D}(B)$ and by $A B$ its product with the domain $\mathcal{D}(A B)=\{x \in \mathcal{D}(B) \mid B x \in \mathcal{D}(A)\}$. Other notation and also basic results from operator theory can be found in [11, Appendix A].

\section{First step: elementary functional calculus}

As we have indicated in $\S 1$, the construction of a functional calculus can be seen as composed of two major steps. The first step consists in constructing an 'elementary' functional calculus while in the second step this 'elementary' calculus is extended to a larger function class. The latter procedure will be axiomatized in the next section. Here we will review a common method for obtaining an elementary calculus.

Given an operator $A$ on a Banach space $X$ one looks for a class $\mathcal{E}$ of functions (defined on the spectrum of $A$ ) and a method

$$
\Phi:(f \mapsto f(A)): \mathcal{E} \longrightarrow \mathcal{L}(X)
$$

of associating a bounded operator $f(A):=\Phi(f)$ with each function $f \in \mathcal{E}$. Since a calculus is for calculation, it seems reasonable to have equations (1.1), i.e. $\mathcal{E}$ should be an algebra and the mapping $\Phi$ should be a homomorphism of algebras. Moreover, the mapping $\Phi$ should have some strong connection with the operator $A$, otherwise our intended notation $f(A)=\Phi(f)$ would not be appropriate. (One could have, for example, $\Phi\left[(\lambda-z)^{-1}\right]=R(\lambda, A)$ for some $\lambda \in \varrho(A)$.)

A common way of obtaining such an elementary calculus is by means of a Cauchy integral:

$$
\Phi(f)=f(A)=\frac{1}{2 \pi \mathrm{i}} \int_{\Gamma} f(z) R(z, A) \mathrm{d} z
$$

with $\Gamma$ positively surrounding $\sigma(A)$ and the representation (1.2) for $a \in \sigma(A)$. If $A$ is not bounded and/or $f$ is not holomorphic on a whole neighbourhood of $\sigma(A)$, then $f$ has to decay nicely near the singular points (according to the growth of $R(\cdot, A)$ ). For such $f$ one can then hope to prove that the definition of $f(A)$ is independent of the chosen $\Gamma$.

We will illustrate these remarks with a well-known example.

Definition 2.1. Let $0 \leqslant \omega<\pi$. An operator $A$ on a Banach space $X$ is called sectorial of angle $\omega$ (in short, $A \in \operatorname{Sect}(\omega)$ ) if $\sigma(A) \subset \overline{S_{\omega}}$ and

$$
M\left(A, \omega^{\prime}\right):=\sup \left\{\|\lambda R(\lambda, A)\| \mid \lambda \notin \overline{S_{\omega^{\prime}}}\right\}<\infty
$$

for all $\omega<\omega^{\prime}<\pi$. The number

$$
\omega_{A}:=\min \{\omega \mid A \in \operatorname{Sect}(\omega)\}
$$

is called the spectral angle (or sectoriality angle) of $A$. 
An operator $A$ on a Banach space $X$ is sectorial of some angle if and only if $(-\infty, 0) \subset$ $\varrho(A)$ and $M(A):=\sup _{t>0}\left\|t(t+A)^{-1}\right\|<\infty$ (see [17, Proposition 1.2.1]). If $A \in \operatorname{Sect}(\omega)$ is injective, then also $A^{-1} \in \operatorname{Sect}(\omega)$. This is due to the fundamental identity

$$
\lambda\left(\lambda+A^{-1}\right)^{-1}=I-\frac{1}{\lambda}\left(\frac{1}{\lambda}+A\right)^{-1},
$$

which holds for all $0 \neq \lambda \in \mathbb{C}$ (in the sense of multivalued operators). For other basic properties of sectorial operators see $[\mathbf{1 7}]$ or $[\mathbf{1 1}]$.

We will construct first an 'elementary' functional calculus for sectorial operators. Let us define

$\mathcal{E}_{0}\left(S_{\varphi}\right):=\left\{f \in H^{\infty}\left(S_{\varphi}\right)\left|\int_{\partial S_{\omega^{\prime}}}\right| f(z) \mid \frac{|\mathrm{d} z|}{|z|}<\infty\right.$ for all $0 \leqslant \omega^{\prime}<\varphi$ and $\left.\lim _{z \rightarrow 0, \infty} f(z)=0\right\}$

and

$$
H_{0}^{\infty}\left(S_{\varphi}\right):=\left\{f \in \mathcal{O}\left(S_{\varphi}\right) \mid \exists C, s>0 \text { such that }|f(z)| \leqslant C \min \left(|z|^{s},|z|^{-s}\right)\right\} .
$$

Obviously, $H_{0}^{\infty}\left(S_{\varphi}\right) \subset \mathcal{E}_{0}\left(S_{\varphi}\right)$ and both are ideals in the algebra $H^{\infty}\left(S_{\varphi}\right)$. If $A \in \operatorname{Sect}(\omega)$, $\omega<\varphi<\pi$ and $f \in \mathcal{E}_{0}\left(S_{\varphi}\right)$, we define

$$
f(A):=\Phi(f):=\frac{1}{2 \pi \mathrm{i}} \int_{\Gamma} f(z) R(z, A) \mathrm{d} z,
$$

where $\Gamma$ is the positively oriented boundary of a sector $S_{\omega^{\prime}}$, with $\omega<\omega^{\prime}<\varphi$ being arbitrary. (Cauchy's theorem shows that $f(A)$ is independent of $\omega^{\prime}$.)

Proposition 2.2. The so-defined mapping $\Phi: \mathcal{E}_{0}\left(S_{\varphi}\right) \longrightarrow \mathcal{L}(X)$ has the following properties.

(i) $\Phi$ is a homomorphism of algebras.

(ii) If $T \in \mathcal{L}(X)$ commutes with the resolvent of $A$, it also commutes with each operator $\Phi(f), f \in \mathcal{E}_{0}\left(S_{\varphi}\right)$.

(iii) For $\lambda \notin \overline{S_{\varphi}}$ and $f \in \mathcal{E}_{0}\left(S_{\varphi}\right)$ we have

$$
R(\lambda, A) \Phi(f)=\Phi(f) R(\lambda, A)=\left(\frac{f(z)}{\lambda-z}\right)(A) .
$$

$$
\left(\frac{z}{(\lambda-z)(\mu-z)}\right)(A)=A R(\lambda, A) R(\mu, A) \text { for } \lambda, \mu \notin \overline{S_{\varphi}} .
$$

Proof. Assertions (i) and (iii) follow from the resolvent identity and Cauchy's theorem. Assertion (ii) is straightforward. To prove (iv), first deform the path in such a way that it avoids 0 , then shift it to the left. Eventually, only the residues in $\lambda$ and $\mu$ will 'survive'. 
As mentioned in $\S 1$ one wants to define $f(A)$ for more general functions $f$ by

$$
f(A):=e(A)^{-1}(e f)(A)
$$

provided $e(A),(e f)(A)$ are already defined and $e(A)$ is injective. Unfortunately, if $A$ is not injective, $e(A) x=0$ for every $e \in \mathcal{E}_{0}\left(S_{\varphi}\right)$ and every $x \in \mathcal{N}(A)$. Hence we cannot proceed in the intended way. To handle this difficulty we enlarge the algebra $\mathcal{E}_{0}\left(S_{\varphi}\right)$ by the function $(1+z)^{-1}$. It is easy to see that

$$
\mathcal{E}\left(S_{\varphi}\right):=\mathcal{E}_{0}\left(S_{\varphi}\right) \oplus \mathbb{C} \frac{1}{1+z}
$$

is in fact an algebra.

We can extend $\Phi$ from $\mathcal{E}_{0}\left(S_{\varphi}\right)$ to $\mathcal{E}\left(S_{\varphi}\right)$ simply by defining

$$
\Phi\left(\frac{1}{1+z}\right):=(1+A)^{-1} .
$$

Then $\Phi: \mathcal{E}\left(S_{\varphi}\right) \longrightarrow \mathcal{L}(X)$ is an algebra homomorphism (one needs (i), (iii) and (iv) of Proposition 2.2 to prove this). We then see that Proposition 2.2 remains valid with $\mathcal{E}_{0}\left(S_{\varphi}\right)$ replaced by $\mathcal{E}\left(S_{\varphi}\right)$. Moreover,

$$
\Phi\left(\frac{1}{\lambda-z}\right)=R(\lambda, A)
$$

for each $\lambda \notin \overline{S_{\varphi}}$.

To sum up (and in more abstract terms), we end up with a commutative algebra $\mathcal{E}:=\mathcal{E}\left(S_{\varphi}\right)$ (without 1!) and an algebra homomorphism $\Phi: \mathcal{E} \longrightarrow \mathcal{L}(X)$ such that $\Phi(f)$ is injective for some $f \in \mathcal{E}$. The algebra $\mathcal{E}$ can be viewed as a subalgebra of the algebra $\mathcal{M}\left(S_{\varphi}\right)$ of all meromorphic functions on the sector $S_{\varphi}$. We now can construct the extension as intended. This will be the content of the following section.

\section{An abstract framework}

In this section we will describe abstractly how to extend a certain basic functional calculus to a wider class of functions. Bearing in mind the situation at the end of the last section, the reader will easily grasp the meaning of the following definition.

Definition 3.1. An abstract functional calculus (AFC) over the Banach space $X$ is a triple $(\mathcal{E}, \mathcal{F}, \bullet)$, where

(i) $\mathcal{F}$ is a commutative algebra with $\mathbf{1}$,

(ii) $\mathcal{E}$ is a subalgebra of $\mathcal{F}$ (without $\mathbf{1}$, in general), and

(iii) $\bullet:\left(e \mapsto e_{\bullet}\right): \mathcal{E} \longrightarrow \mathcal{L}(X)$ is a homomorphism of algebras.

We say that the AFC is non-degenerate or proper, if the set

$$
\left\{e \in \mathcal{E} \mid e_{\bullet} \text { is injective }\right\}
$$

is not empty. 
Take $f \in \mathcal{F}$. If there is $e \in \mathcal{E}$ such that $e f \in \mathcal{E}$ and $e_{\bullet}$ is injective, we call $f$ regularizable by $\mathcal{E}$ and $e$ a regularizer for $f$. In this case we define

$$
f_{\bullet}:=\left(e_{\bullet}\right)^{-1}(e f) \bullet
$$

Lemma 3.2. The definition (3.1) yields a closed operator on $X$ and is independent of the regularizer $e$.

Proof. Let $h \in \mathcal{E}$ be a second regularizer for $f$ and define $A:=\left(e_{\bullet}\right)^{-1}(e f)$. and $B:=\left(h_{\bullet}\right)^{-1}(h f)_{\bullet}$. Because $e_{\bullet} h_{\bullet}=(e h)_{\bullet}=(h e) \bullet=h_{\bullet} e_{\bullet}$, we have $\left(e_{\bullet}\right)^{-1}\left(h_{\bullet}\right)^{-1}=$ $\left(h_{\bullet}\right)^{-1}\left(e_{\bullet}\right)^{-1}$. Now it follows that

$$
\begin{aligned}
A & =\left(e_{\bullet}\right)^{-1}(e f) \bullet=\left(e_{\bullet}\right)^{-1}\left(h_{\bullet}\right)^{-1} h_{\bullet}(e f) \bullet=(h \bullet)^{-1}\left(e_{\bullet}\right)^{-1}(h e f) \\
& =\left(h_{\bullet}\right)^{-1}\left(e_{\bullet}\right)^{-1} e_{\bullet}(h f) \bullet=\left(h_{\bullet}\right)^{-1}(h f) \bullet=B .
\end{aligned}
$$

Note that $\mathbf{1}$ is regularizable if and only if the AFC is proper. In this case

$$
\mathcal{F}_{r}:=\{f \in \mathcal{F} \mid f \text { is regularizable }\}
$$

clearly is a subalgebra of $\mathcal{F}$ which contains $\mathcal{E}$. Moreover, we have extended the original representation $\mathcal{E} \longrightarrow \mathcal{L}(X)$ to a map

$$
\left(f \mapsto f_{\bullet}\right): \mathcal{F}_{r} \longrightarrow\{\text { closed operators on } X\} .
$$

(Since the AFC is proper, $\mathcal{E} \subset \mathcal{F}_{r}$. If $e, f \in \mathcal{E}$ with $e_{\bullet}$ injective, one has $\left(e_{\bullet}\right)^{-1}(e f) \bullet$ $\left(e_{\bullet}\right)^{-1} e_{\bullet} f_{\bullet}=f_{\bullet}$. This shows that the map on $\mathcal{F}_{r}$ is indeed an extension of the original.)

Sometimes we will call the original mapping $\bullet: \mathcal{E} \longrightarrow \mathcal{L}(X)$ the primary (functional) calculus (PFC) and the extension defined above the extended (functional) calculus. The algebra $\mathcal{F}_{r}$ is called the domain of the $\operatorname{AFC}(\mathcal{E}, \mathcal{F}, \bullet)$. We collect some basic properties.

Proposition 3.3. Let $(\mathcal{E}, \mathcal{F}, \bullet)$ be a proper abstract functional calculus over the Banach space $X$. Then the following assertions hold.

(i) If $T \in \mathcal{L}(X)$ commutes with each $e_{\bullet}, e \in \mathcal{E}$, then it commutes with each $f_{\bullet}, f \in \mathcal{F}_{r}$.

(ii) One has $\mathbf{1} \in \mathcal{F}_{r}$ and $\mathbf{1} \bullet=I$.

(iii) Given $f, g \in \mathcal{F}_{r}$, one has

$$
\begin{aligned}
f_{\bullet}+g \bullet & \subset(f+g) \bullet \\
f_{\bullet} g_{\bullet} & \subset(f g)_{\bullet},
\end{aligned}
$$

with $\mathcal{D}\left(f_{\bullet} g_{\bullet}\right)=\mathcal{D}((f g) \bullet) \cap \mathcal{D}\left(g_{\bullet}\right)$.

(iv) If $f, g \in \mathcal{F}_{r}$ such that $f g=\mathbf{1}$, then $f_{\bullet}$ is injective with $\left(f_{\bullet}\right)^{-1}=g_{\bullet}$. 
(v) Let $f \in \mathcal{F}_{r}$ and $F$ be a subspace of $\mathcal{D}\left(f_{\bullet}\right)$. Assume that there is a sequence $\left(e_{n}\right)_{n} \subset \mathcal{E}$ such that $e_{n} \bullet \rightarrow I$ strongly as $n \rightarrow \infty$ and $\mathcal{R}\left(e_{n} \bullet\right) \subset F$ for all $n \in \mathbb{N}$. Then $F$ is a core for $f_{\bullet}$.

Proof. (i). Let $T \in \mathcal{L}(X)$ commute with every $e_{\bullet}, e \in \mathcal{E}$. Take $f \in \mathcal{F}_{r}$ and a regularizer $e \in \mathcal{E}$ for $f$. Then we have

$$
T f_{\bullet}=T\left(e_{\bullet}\right)^{-1}(e f) \bullet \subset\left(e_{\bullet}\right)^{-1} T(e f) \bullet=\left(e_{\bullet}\right)^{-1}(e f) \bullet T=f_{\bullet} T .
$$

(ii). Since the AFC is proper, there exists $e \in \mathcal{E}$ such that $e_{\bullet}$ is injective. Clearly, $e$ regularizes $\mathbf{1}$. Moreover, we have $\mathbf{1}_{\bullet}=e_{\bullet}^{-1}(e \mathbf{1}) \bullet=e_{\bullet}^{-1} e_{\bullet}=I$.

(iii). Take $f, g \in \mathcal{F}_{r}$ and let $e_{1}, e_{2} \in \mathcal{E}$ be regularizers for $f, g$, respectively. Then $e:=e_{1} e_{2}$ is a regularizer for both $f$ and $g$, hence for $f+g$. Also efg $=\left(e_{1} f\right)\left(e_{2} g\right) \in \mathcal{E}$, whence $e$ is also a regularizer for $f g$. We have

$$
\begin{aligned}
f_{\bullet}+g_{\bullet} & =e_{\bullet}^{-1}(e f)_{\bullet}+e_{\bullet}^{-1}(e g) \bullet \subset e_{\bullet}^{-1}\left[(e f) \bullet+(e g)_{\bullet}\right] \\
& =e_{\bullet}^{-1}(e(f+g))_{\bullet}=(f+g) \bullet
\end{aligned}
$$

and

$$
\begin{aligned}
f \bullet g \bullet & =e_{1 \bullet}^{-1}\left(e_{1} f\right) \bullet e_{2 \bullet}^{-1}\left(e_{2} g\right) \bullet \subset e_{1 \bullet}^{-1} e_{2 \bullet}^{-1}\left(e_{1} f\right) \bullet\left(e_{2} g\right) \bullet \\
& =\left[e_{2} \bullet e_{1} \bullet\right]^{-1}(e f g) \bullet=e_{\bullet}^{-1}(e f g) \bullet=(f g) \bullet .
\end{aligned}
$$

To prove the assertions concerning the domains, let $x \in \mathcal{D}((f g) \bullet) \cap \mathcal{D}\left(g_{\bullet}\right)$. Note that since $\left(e_{1} f\right)$. commutes with $e_{2}$. it also commutes with $e_{2 \bullet}^{-1}$. By assumption, $y:=\left(e_{2} g\right) \bullet x \in \mathcal{D}\left(e_{2 \bullet}^{-1}\right)$, whence also $\left(e_{1} f\right) \bullet y \in \mathcal{D}\left(e_{2 \bullet}^{-1}\right)$ and

$$
\left(e_{1} f\right) \bullet g \bullet x=\left(e_{1} f\right) \bullet e_{2 \bullet}^{-1} y=e_{2 \bullet}^{-1}\left(e_{1} f\right) \bullet y=e_{2 \bullet}^{-1}(e f g) \bullet x \in \mathcal{D}\left(e_{1}^{-1}\right)
$$

by assumption and the identity $e_{\bullet}^{-1}=e_{1}^{-1} e_{2 \bullet}^{-1}$. Consequently, $g_{\bullet} x \in \mathcal{D}\left(f_{\bullet}\right)$, whence $x \in \mathcal{D}\left(f_{\bullet} g_{\bullet}\right)$.

(iv). Assume $f, g \in \mathcal{F}_{r}$ with $f g=\mathbf{1}$. By (ii) and (iii) we have $g_{\bullet} f_{\bullet} \subset(f g)_{\bullet}=\mathbf{1}_{\bullet}=I$ and $\mathcal{D}\left(g_{\bullet} f_{\bullet}\right)=\mathcal{D}(I) \cap \mathcal{D}\left(f_{\bullet}\right)=\mathcal{D}\left(f_{\bullet}\right)$. Interchanging $f$ and $g$ proves the statement.

$(\mathrm{v})$. Let $x \in \mathcal{D}\left(f_{\bullet}\right)$ and define $y:=f_{\bullet} x$. With $x_{n}:=e_{n} \bullet x$ and $y_{n}:=e_{n} \bullet y$ we have $x_{n} \rightarrow x, x_{n} \in F$ and $f_{\bullet} x_{n}=f_{\bullet} e_{n} \bullet x=e_{n} \bullet f_{\bullet} x=y_{n} \rightarrow y$.

In general one cannot expect equality in (iii) of Proposition 3.3. However, if we define

$$
\mathcal{F}_{b}:=\left\{f \in \mathcal{F}_{r} \mid f_{\bullet} \in \mathcal{L}(X)\right\},
$$

we obtain the following.

Corollary 3.4. Let $\mathcal{E}, \mathcal{F}, X$ be as above.

(i) For $f \in \mathcal{F}_{r}, g \in \mathcal{F}_{b}$ the identities

$$
f_{\bullet}+g_{\bullet}=(f+g) \bullet \quad \text { and } \quad f_{\bullet} g_{\bullet}=(f g) \bullet
$$

hold. 
(ii) The set $\mathcal{F}_{b}$ is a subalgebra with $\mathbf{1}$ of $\mathcal{F}$ and the map

$$
\left(f \mapsto f_{\bullet}\right): \mathcal{F}_{b} \longrightarrow \mathcal{L}(X)
$$

is a homomorphism of algebras with $\mathbf{1}$.

(iii) If $f \in \mathcal{F}_{b}$ is such that $f_{\bullet}$ is injective, then

$$
\left(f_{\bullet}\right)^{-1} g_{\bullet} f_{\bullet}=g_{\bullet}
$$

holds for all $g \in \mathcal{F}_{r}$.

Proof. (i). By (iii) of Proposition 3.3 we have $f_{\bullet}+g_{\bullet} \subset(f+g) \bullet$ and $(f+g)_{\bullet}-g_{\bullet} \subset$ $(f+g-g)_{\bullet}=f_{\bullet}$. Since $g_{\bullet}$ is assumed to be bounded, $\mathcal{D}((f+g) \bullet)=\mathcal{D}\left(f_{\bullet}\right)$. This readily implies $f_{\bullet}+g_{\bullet}=(f+g)$. The second assertion is immediate from (iii) of Proposition 3.3 since we have $\mathcal{D}\left(g_{\bullet}\right)=X$.

The statement in (ii) follows from (i).

(iii). We can find $e \in \mathcal{E}$ which regularizes both $f$ and $g$. Now we compute

$$
f_{\bullet}^{-1} g_{\bullet} f_{\bullet}=f_{\bullet}^{-1} e_{\bullet}^{-1}(e g)_{\bullet} f_{\bullet}=f_{\bullet}^{-1} e_{\bullet}^{-1} f_{\bullet}(e g)_{\bullet}=e_{\bullet}^{-1} f_{\bullet}^{-1} f_{\bullet}(e g)_{\bullet}=e_{\bullet}^{-1}(e g) \bullet=g_{\bullet}
$$

Here we used (ii) and the identity $e_{\bullet}^{-1} f_{\bullet}^{-1}=f_{\bullet}^{-1} e_{\bullet}^{-1}$, which is true since $e_{\bullet}$ and $f_{\bullet}$ are both bounded and injective.

Using this new information we can improve on (iv) of Proposition 3.3.

Corollary 3.5. Let $\mathcal{E}, \mathcal{F}, X$ be as above. Suppose $f \in \mathcal{F}_{r}, g \in \mathcal{F}$ such that $f g=1$. Then

$$
g \in \mathcal{F}_{r} \Longleftrightarrow f_{\bullet} \text { is injective. }
$$

In this case, we have $g_{\bullet}=f_{\bullet}^{-1}$.

Proof. One direction of the biimplication is simply (iv) of Proposition 3.3. Assume that $f_{\bullet}$ is injective and let $e \in \mathcal{E}$ be a regularizer for $f$. Then $f e \in \mathcal{E},(f e) g=e \in \mathcal{E}$ and $(f e)_{\bullet}=f_{\bullet} e_{\bullet}$ is injective. This means that $f e$ is a regularizer for $g$ !

Let us illustrate the results from above by looking at our principal example. Namely, let $A \in \operatorname{Sect}(\omega)$ be a sectorial operator on a Banach space $X$ and consider the $(\mathcal{E}, \mathcal{F}, \Phi)$, where $\mathcal{E}:=\mathcal{E}\left(S_{\varphi}\right), \mathcal{F}:=\mathcal{M}\left(S_{\varphi}\right)$, and $\Phi$ is the mapping constructed in $\S 2$. We already know that this yields a proper AFC. Let us write $\mathcal{M}\left(S_{\varphi}\right)_{A}:=\mathcal{F}_{r}$ for the regularizable functions and $H(A):=\mathcal{F}_{b}$ for those which give rise to bounded operators. Moreover, we write as usual $f(A)$ instead of $\Phi(f)$ in this context. Then we obtain the following theorem.

Theorem 3.6. Let $0 \leqslant \omega<\varphi<\pi, A \in \operatorname{Sect}(\omega)$ and $f \in \mathcal{M}\left(S_{\varphi}\right)_{A}$. Then the following assertions hold.

(i) If $T \in \mathcal{L}(X)$ commutes with $A$, it also commutes with $f(A)$. If $f(A) \in \mathcal{L}(X)$, then $f(A)$ commutes with $A$. 
(ii) $\mathbf{1}(A)=I$ and $(z)(A)=A$.

(iii) Let also $g \in \mathcal{M}\left(S_{\varphi}\right)_{A}$. Then

$$
f(A)+g(A) \subset(f+g)(A) \quad \text { and } \quad f(A) g(A) \subset(f g)(A) .
$$

Furthermore, $\mathcal{D}((f g)(A)) \cap \mathcal{D}(g(A))=\mathcal{D}(f(A) g(A))$ and one has equality in the above relations if $g \in H(A)$.

(iv) The mapping $(f \mapsto f(A)): H(A) \longrightarrow \mathcal{L}(X)$ is a homomorphism of algebras.

(v) One has $f(A)=g(A)^{-1} f(A) g(A)$ if $g \in H(A)$ and $g(A)$ is injective.

(vi) Let $\lambda \in \mathbb{C}$. Then

$$
\frac{1}{\lambda-f(z)} \in \mathcal{M}\left(S_{\varphi}\right)_{A} \Longleftrightarrow \lambda-f(A) \text { is injective. }
$$

In this case $(\lambda-f(z))^{-1}(A)=(\lambda-f(A))^{-1}$. In particular, $\lambda \in \varrho(f(A))$ if and only if $(\lambda-f(z))^{-1} \in H(A)$.

Proof. This is more or less a restatement of Proposition 3.3 and Corollaries 3.4 and 3.5. For (ii) note that $(1+z)^{-2}$ regularizes $z$, whence $(z)(A)=(1+A)^{2}\left(z(1+z)^{-2}\right)(A)=$ $(1+A)^{2} A(1+A)^{-2}=A$ by Proposition 2.2 .

With the help of Theorem 3.6 it can be shown that the symbol $r(A)$ with $r$ being a rational function with poles in the right half-plane has its usual meaning (cf. [11]).

We return to the abstract treatment. Suppose $(\mathcal{E}, \mathcal{F}, \bullet)$ is a proper AFC over the Banach space $X$. Call a subalgebra $\mathcal{D} \subset \mathcal{F}_{b}$ admissible if the set $\left\{f \in \mathcal{D} \mid f_{\bullet}\right.$ is injective $\}$ is not empty. In this case $(\mathcal{D}, \mathcal{F})$ is another proper AFC. Let us denote

$$
\langle\mathcal{D}\rangle:=\left\{f \in \mathcal{F} \mid \text { there is } d \in \mathcal{D} \text { such that } d f \in \mathcal{D} \text { and } d_{\bullet} \text { is injective }\right\}
$$

the regularizable elements of this AFC. We call $\langle\mathcal{D}\rangle$ the domain generated by $\mathcal{D}$ and $\mathcal{D}$ its generator. In this terminology, $\mathcal{F}_{r}=\langle\mathcal{E}\rangle$. The next proposition shows in particular that $\langle\mathcal{D}\rangle$ is always a subalgebra of $\mathcal{F}_{r}$ whence nothing is gained by passing from $\mathcal{E}$ to $\mathcal{F}_{b}$.

Proposition 3.7. Let $(\mathcal{E}, \mathcal{F}, \bullet)$ be a proper $A F C$ on the Banach space $X$ and let $\mathcal{D}$ be an admissible subalgebra of $\mathcal{F}_{b}$. If $f \in \mathcal{F}$ and $g \in\langle\mathcal{D}\rangle$ such that $g_{\bullet}$ is injective and $g f \in\langle\mathcal{D}\rangle$, then already $f \in\langle\mathcal{D}\rangle$.

Proof. By assumption there are regularizing elements $d_{1}, d_{2} \in \mathcal{D}$ for $g, f g$, respectively. Letting $d:=d_{1} d_{2}$ we see that $d$ regularizes both $g$ and $f g$. Since $\left(d_{\bullet}\right)^{-1}(d g) \bullet=g \bullet$ is injective, the operator $(d g)$ • also is. Hence, $d g$ regularizes $f$, whence $f \in\langle\mathcal{D}\rangle$.

A generator of the AFC is an admissible subalgebra $\mathcal{D}$ such that $\langle\mathcal{D}\rangle=\mathcal{F}_{r}$.

Corollary 3.8. Let $(\mathcal{E}, \mathcal{F}, \bullet)$ be a proper AFC over the Banach space $X$. Let $\mathcal{D}, \mathcal{D}^{\prime}$ be admissible subalgebras of $\mathcal{F}_{b}$ such that $\mathcal{D}^{\prime} \subset\langle\mathcal{D}\rangle$. Then $\left\langle\mathcal{D}^{\prime}\right\rangle \subset\langle\mathcal{D}\rangle$. In particular, an admissible subalgebra $\mathcal{D}$ of $\mathcal{F}_{b}$ is a generator of the $A F C$ if and only if $\mathcal{E} \subset\langle\mathcal{D}\rangle$. 
Proof. This follows immediately from Proposition 3.7.

As an application, consider the case of a sectorial operator $A$. If $A$ is not injective, the AFC $\left(H_{0}^{\infty}\left(S_{\varphi}\right), \mathcal{M}\left(S_{\varphi}\right), \Phi\right)$ is not proper. However, if $A$ is injective, then $H_{0}^{\infty}\left(S_{\varphi}\right)$ is in fact a generator of the $\operatorname{AFC}\left(\mathcal{E}\left(S_{\varphi}\right), \mathcal{M}\left(S_{\varphi}\right), \Phi\right)$.

It should be clear that abstract functional calculi over the Banach space $X$ are the objects of a category. We describe the morphisms of this category. Let $(\mathcal{E}, \mathcal{F}, \bullet)$ and $\left(\mathcal{E}^{\prime}, \mathcal{F}^{\prime}, \bullet\right)$ be proper AFCs over the Banach space $X$. A morphism

$$
\Psi:(\mathcal{E}, \mathcal{F}, \bullet) \longrightarrow\left(\mathcal{E}^{\prime}, \mathcal{F}^{\prime}, \bullet\right)
$$

between these AFCs consists of a homomorphism of algebras $\Psi: \mathcal{F} \longrightarrow \mathcal{F}^{\prime}$ with $\Psi(\mathcal{E}) \subset \mathcal{E}^{\prime}$ and $\Psi(e)_{\bullet}=e_{\bullet}$ for all $e \in \mathcal{E}$. Not surprisingly, the extension procedure is functorial, as the next proposition shows.

Proposition 3.9. Let $\Psi:(\mathcal{E}, \mathcal{F}, \bullet) \longrightarrow\left(\mathcal{E}^{\prime}, \mathcal{F}^{\prime}, \bullet\right)$ be a morphism of proper AFCs on the Banach space $X$. Then $\Psi\left(\mathcal{F}_{r}\right) \subset\left(\mathcal{F}^{\prime}\right)_{r}$ with $\Psi(f) \bullet=f \bullet$ for every $f \in \mathcal{F}_{r}$.

Proof. Let $f \in \mathcal{F}_{r}$ and let $e \in \mathcal{E}$ be a regularizer for $f$. Since $\Psi(e)_{\bullet}=e_{\bullet}$ is injective and $\Psi(e) \Psi(f)=\Psi(e f) \in \Psi(\mathcal{E}) \subset \mathcal{E}^{\prime}$, the element $\Psi(e)$ is a regularizer for $\Psi(f)$. Moreover, $\Psi(f) \bullet=\left[\Psi(e)_{\bullet}\right]^{-1}[\Psi(e) \Psi(f)]_{\bullet}=\left(e_{\bullet}\right)^{-1} \Psi(e f) \bullet=\left(e_{\bullet}\right)^{-1}(e f)_{\bullet}=f_{\bullet}$.

Remark 3.10. The above lemma may seem, at first glance, to be another bit of abstract nonsense. However, it is of great use, mostly in the case when $\mathcal{F}^{\prime}$ is in fact a superalgebra of $\mathcal{F}$ and $\Psi$ is just the inclusion mapping. In such a situation Proposition 3.9 implies that for a consistent extension of an AFC one has to take care only of the primary calculus, whereas consistency of the extended calculus is then automatic.

We give some applications. Take a sectorial operator $A$ as in $\S 2$ with the additional condition that $0 \in \varrho(A)$. Then functions need not be defined on the whole of $S_{\varphi}$ but may be undefined on a small ball $B$ round 0 . Our original class $\mathcal{F}=\mathcal{M}\left(S_{\varphi}\right)$ can be embedded into $\mathcal{F}^{\prime}:=\mathcal{M}\left(S_{\varphi} \backslash B\right)$ and the primary calculus $\Phi$ defined on $\mathcal{E}\left(S_{\varphi}\right)$ can be consistently extended to those $f \in H^{\infty}\left(S_{\varphi} \backslash B\right)$ which decay 'sufficiently fast' at infinity, in order to make the corresponding Cauchy-integral converge. The mapping ' $\Psi$ ' from Proposition 3.9 is the just ordinary inclusion.

A similar extension is possible if $A$ is sectorial and bounded. Then functions need not be defined near $\infty$. As a matter of fact, one can think of other spectral properties (like separation of the spectrum into disjoint closed subsets) which lead to other kinds of extensions.

Let us turn to another application. Let $A \in \operatorname{Sect}(\omega)$ as in $\S 2$. Then up to now we have a proper $\operatorname{AFC}\left(\mathcal{E}\left(S_{\varphi}\right), \mathcal{M}\left(S_{\varphi}\right), \Phi\right)$ for each $\omega<\varphi<\pi$. Let us write $\Phi=\Phi_{\varphi}$ for the moment to emphasize dependence on $\varphi$. Now, since all functions are meromorphic, we have natural inclusions

$$
\mathcal{E}_{0}\left(S_{\varphi}\right) \hookrightarrow \mathcal{E}_{0}\left(S_{\psi}\right) \quad \text { and } \quad \mathcal{M}\left(S_{\varphi}\right) \hookrightarrow \mathcal{M}\left(S_{\psi}\right)
$$

for $(\omega<\psi<\varphi<\pi)$. Clearly, $\left.\Phi_{\psi}\right|_{\mathcal{E}\left(S_{\varphi}\right)}=\Phi_{\varphi}$, whence Proposition 3.9 is applicable. 
Finally, we can form the inductive limits

$$
\mathcal{E}\left[S_{\omega}\right]:=\bigcup_{\omega<\varphi<\pi} \mathcal{E}\left(S_{\varphi}\right) \quad \text { and } \quad \mathcal{M}\left[S_{\omega}\right]:=\bigcup_{\omega<\varphi<\pi} \mathcal{M}\left(S_{\varphi}\right)
$$

and obtain a proper $\operatorname{AFC}\left(\mathcal{E}\left[S_{\omega}\right], \mathcal{M}\left[S_{\omega}\right], \Phi\right)$. Clearly, we have

$$
\mathcal{M}\left[S_{\omega}\right]_{A}=\bigcup_{\omega<\varphi<\pi} \mathcal{M}\left(S_{\varphi}\right)_{A} .
$$

(We refrain from proving an abstract result on inductive limits of AFCs here.)

\section{Regularizable functions}

Whereas the construction of an elementary functional calculus is usually done for certain classes of operators (like we did with sectorial operators), the extension procedure described in the last section takes into account individual (spectral) properties of the given operator. Here a difficulty arises. Namely, in general it is not so clear which elements $e \in \mathcal{E}\left[S_{\omega}\right]$ give rise to injective operators $e(A)$, i.e. which can serve as regularizers. Thus the domain $\mathcal{M}\left[S_{\omega}\right]_{A}$ in general will be unknown. Therefore, it is of considerable interest to specify subclasses of $\mathcal{M}\left[S_{\omega}\right]_{A}$. The first is a negative result.

Lemma 4.1. Let $A \in \operatorname{Sect}(\omega)$ and $\omega<\varphi<\pi$. Let $e \in \mathcal{E}\left(S_{\varphi}\right)$ and $\lambda \in \sigma_{p}(A)$ with $e(\lambda)=0$. Then $e(A)$ is not injective.

Proof. Suppose first that $\lambda \neq 0$. Then

$$
h:=\frac{e}{\lambda-z} \in \mathcal{E}\left(S_{\varphi}\right)
$$

again, whence $e(A) \supset h(A)(\lambda-A)$. If $\lambda=0$, then $e \in \mathcal{E}_{0}\left(S_{\varphi}\right)$ and $e(A) x=0$ for every $x \in \mathcal{N}(A)$ by direct computation (cf. [12, Proposition 3.1]).

The next result gives a necessary condition for a function $f \in \mathcal{M}\left(S_{\varphi}\right)$ to be regularizable in case $A$ is not injective.

Lemma 4.2. Let $A \in \operatorname{Sect}(\omega)$ with $\mathcal{N}(A) \neq 0$. Take $\varphi>\omega$ and $f \in \mathcal{M}\left(S_{\varphi}\right)_{A}$. Then there is $c \in \mathbb{C}$ such that

$$
\int_{\partial S_{\omega^{\prime}},|z| \leqslant \varepsilon}|f(z)-c| \frac{|\mathrm{d} z|}{|z|}<\infty \quad\left(0 \leqslant \omega^{\prime}<\varphi\right) .
$$

Proof. Let $e \in \mathcal{E}\left(S_{\varphi}\right)$ be a regularizer for $f$, i.e. ef $\in \mathcal{E}\left(S_{\varphi}\right)$ and $e(A)$ is injective. Since $e(A) x=e(0) x$ for all $x \in \mathcal{N}(A), e(0) \neq 0$. This clearly implies that $f$ has a finite limit $c:=f(0)$ at 0 and

$$
f(z)-f(0)=\frac{1}{e(z)}[(e f)(z)-(e f)(0)-f(0)(e(z)-e(0))]
$$

whence the assertion follows. 
The last lemma leads one to expect that non-injective operators $A$ need special treatment in many situations. In particular in the proof of the composition rule in $\S 7$ noninjectivity of $A$ forces one to consider a restricted calculus, i.e. functions with a stronger regularity at 0 are needed. Define

$$
\mathcal{E}_{\text {res }}\left(S_{\varphi}\right):=H_{0}^{\infty}\left(S_{\varphi}\right) \oplus \mathbb{C} \frac{1}{1+z} .
$$

which is a subalgebra of $\mathcal{E}\left(S_{\varphi}\right)$, and define $\mathcal{E}_{\text {res }}\left[S_{\omega}\right]=\bigcup_{\omega<\varphi<\pi} \mathcal{E}_{\text {res }}\left(S_{\varphi}\right)$. Consider the AFC $\left(\mathcal{E}_{\text {res }}\left[S_{\omega}\right], \mathcal{M}\left[S_{\omega}\right], \Phi\right)$, which we will call the restricted calculus in the sequel. If $A$ is injective, the domain of this restricted calculus (i.e. the set of regularizable functions) is the same as for the $\operatorname{AFC}\left(\mathcal{E}\left[S_{\omega}\right], \mathcal{M}\left[S_{\omega}\right], \Phi\right)$, since then $H_{0}^{\infty}$ is already a generator (cf. $\S 3$ ). However, if $A$ is not injective, one obtains indeed a smaller calculus, as the following proposition shows.

Proposition 4.3. Let $0<\varphi<\pi$ and $f \in \mathcal{O}\left(S_{\varphi}\right)$. Then the following assertions are equivalent:

(i) $f \in \mathcal{E}_{\text {res }}\left(S_{\varphi}\right)$;

(ii) $f \in H^{\infty}\left(S_{\varphi}\right)$ and there are $c \in \mathbb{C}, \alpha>0$ such that

$$
\begin{aligned}
f(z) & =O\left(|z|^{-\alpha}\right) & & \left(z \in S_{\varphi}, z \rightarrow \infty\right), \\
f(z)-c & =O\left(|z|^{\alpha}\right) & & \left(z \in S_{\varphi}, z \rightarrow 0\right) .
\end{aligned}
$$

Let $A \in \operatorname{Sect}(\omega)$ with $\omega<\varphi$ and $\mathcal{N}(A) \neq 0$. For $f \in \mathcal{M}\left(S_{\varphi}\right)$ regularizable by $\mathcal{E}_{\text {res }}\left(S_{\varphi}\right)$ there is $c \in \mathbb{C}$ and $\alpha>0$ such that

$$
f(z)-c=O\left(|z|^{\alpha}\right) \quad\left(z \in S_{\varphi}, z \rightarrow 0\right) .
$$

Proof. The first part is straightforward. Let $e \in \mathcal{E}_{\text {res }}\left(S_{\varphi}\right)$ be a regularizer for $f$, i.e. ef $\in \mathcal{E}_{\text {res }}\left(S_{\varphi}\right)$ and $e(A)$ is injective. Since $e(A) x=e(0) x$ for all $x \in \mathcal{N}(A), e(0) \neq 0$. Hence, by the first part of the proposition, $f$ has a finite limit $c:=f(0)$ at 0 and

$$
f(z)-f(0)=\frac{1}{e(z)}[(e f)(z)-(e f)(0)+f(0)(e(z)-e(0))]
$$

whence the assertion follows.

In the sequel we will use the abbreviation

$$
\mathcal{M}\left[S_{\omega}\right]_{\mathrm{res}, A}
$$

to denote the functions $f \in \mathcal{M}\left[S_{\omega}\right]$ which are regularizable within the restricted calculus $\left(\mathcal{E}_{\text {res }}\left[S_{\omega}\right], \mathcal{M}\left[S_{\omega}\right], \Phi\right)$.

It is time to present some positive results. Since resolvents are injective operators, powers of $(1+z)^{-1}$ are always good regularizers. This shows that holomorphic functions with at most polynomial growth at $\infty$ and having the limit property at 0 which appears in 
Lemma 4.2 are always regularizable even within the restricted calculus. If $A$ is injective, powers of $z(1+z)^{-2}$ regularize every function with at most polynomial growth at $\infty$ and 0 . This is well known in the literature (see $[\mathbf{1}]$ ).

However, we can do much better, even in the non-injective case.

Lemma 4.4. Let $A \in \operatorname{Sect}(\omega), \omega<\varphi<\pi$ and $\alpha>0$ such that $\alpha \varphi<\pi / 2$. Then

$$
f(z):=\mathrm{e}^{-t z^{\alpha}} \in \mathcal{E}_{\mathrm{res}}\left(S_{\varphi}\right) \text { and } f(A) \text { is injective }
$$

for every $t>0$.

Proof. The first assertion follows from Lemma 4.3. To prove the second, we reduce the problem to the case $\varphi<\pi / 2, \alpha=1$. Namely, setting $B:=A^{\alpha}$ it is known that $B \in \operatorname{Sect}(\alpha \omega)$ (see [17] or [11]). Applying the composition rule (see Theorem 7.1 below) we obtain $f(A)=\mathrm{e}^{-t z}(B)=: \mathrm{e}^{-t B}$.

One can now show that $\left(\mathrm{e}^{-t B}\right)_{t>0}$ is a uniformly bounded, holomorphic semigroup (see $[\mathbf{1 1}$, Chapter II] or [15]). By means of the Fubini theorem one shows that the representation

$$
\frac{1}{1+z}=\int_{0}^{\infty} \mathrm{e}^{-t} \mathrm{e}^{-t z} \mathrm{~d} t
$$

carries over to operators: $(1+B)^{-1}=\int_{0}^{\infty} \mathrm{e}^{-t} \mathrm{e}^{-t B} \mathrm{~d} t$. Now, if $x \in \mathcal{N}\left(\mathrm{e}^{-t B}\right)$ for some $t>0$, this holds even for all $t>0$, by the semigroup property and holomorphy. Hence $(1+B)^{-1} x=0$, and therefore $x=0$.

Proposition 4.5. Let $A \in \operatorname{Sect}(\omega), \omega<\varphi<\pi$ and $f \in \mathcal{O}\left(S_{\varphi}\right)$. Suppose the two following conditions are satisfied.

(i) There exist $\beta>0$ and $c \in \mathbb{C}$ such that $f(z)-c=O\left(|z|^{\beta}\right)$ for $z \rightarrow 0$.

(ii) There exist $\alpha, t>0$ with $\alpha \varphi<\pi / 2$ and $f(z)=O\left(\mathrm{e}^{t \operatorname{Re} z^{\alpha}}\right)$ for $z \rightarrow \infty$.

Then $f \in \mathcal{M}\left(S_{\varphi}\right)_{\text {res, } A}$, i.e. $f(A)$ is defined within the restricted calculus.

The same conclusion holds if $A$ is injective and both $f$ and $f(1 / z)$ satisfy condition (ii).

Proof. The first part follows easily from Lemma 4.4 and Lemma 4.3 using as regularizer the function $e(z):=\mathrm{e}^{-s z^{\alpha}}$ with $s>t$. When $A$ is injective one can use a regularizer of the form $e(z):=\mathrm{e}^{-s_{1} z^{\alpha_{1}}-s_{2} z^{-\alpha_{2}}}$ (and use Corollary 7.5).

\section{Algebraic versus topological extension}

We want to emphasize the fact that our abstract extension procedure from $\S 3$ is purely algebraic. The topology is hidden in the construction of the primary calculus (e.g. by means of a Cauchy integral as in our concrete example). Nevertheless, one could think of extending the primary calculus to a larger primary calculus by topological means, according to the following pattern:

$$
\text { if }\left(e_{n}\right)_{n} \subset \mathcal{E}, f \in \mathcal{H}, e_{n} \rightarrow f \text { and } e_{n} \bullet \rightarrow T \text {, then define } f_{\bullet}:=T \text {. }
$$


Here we suppose that there is a subalgebra $\mathcal{H} \subset \mathcal{F}$ suitably topologized and with $\mathcal{E} \subset \mathcal{H}$. Moreover, we fix any reasonable topology on $\mathcal{L}(X)$ (e.g. the strong operator topology) in order to give meaning to the convergence $e_{n} \bullet T$.

A moment's reflection shows that, for Definition (5.1) to be reasonable, the following axiom is required:

$$
\text { (Top) : } \quad e_{n} \rightarrow 0, e_{n} \in \mathcal{E} \quad \Longrightarrow \quad e_{n} \rightarrow 0 .
$$

Given this, one then obtains via Definition (5.1) a consistent extension of the original $\operatorname{AFC}(\mathcal{E}, \mathcal{F}, \bullet)$ to the larger $\operatorname{AFC}(\tilde{\mathcal{E}}, \mathcal{F}, \bullet)$, where

$$
\tilde{\mathcal{E}}:=\left\{f \in \mathcal{H} \mid \exists\left(e_{n}\right)_{n} \subset \mathcal{E}, e_{n} \rightarrow f, T:=\lim _{n} e_{n} \bullet \text { exists }\right\} .
$$

In the following we want to examine if such a procedure can be (and should be) done in our model case, the functional calculus for sectorial operators.

Let us first say some words on the topology which could be reasonably used. Let $A$ be a sectorial operator on a reflexive space $X$ with non-trivial kernel $\mathcal{N}(A) \neq 0$, and let $P$ be the projection onto $\overline{\mathcal{R}(A)}$ along $\mathcal{N}(A)$. Defining $f_{n}:=n z /(1+n z)$ we have $f_{n} \rightarrow \mathbf{1}$ pointwise and the sequence $\left(f_{n}\right)_{n}$ is uniformly bounded on each sector $S_{\varphi}$ with $\varphi<\pi$. However, $f_{n}(A) \rightarrow P \neq I$ strongly. Hence boundedly pointwise convergence is not a good topology to use for the extension.

The following proposition shows that uniform convergence on sectors $S_{\varphi}$ with $\omega<\varphi$ is a possible choice. We will see in a moment that this indeed leads to a really larger calculus if $A$ is not injective.

Proposition 5.1. Let $A \in \operatorname{Sect}(\omega), \omega<\varphi<\pi$, $\left(e_{n}\right)_{n} \subset \mathcal{E}_{\text {res }}\left(S_{\varphi}\right)$ with $e_{n} \rightarrow 0$ uniformly on $S_{\varphi}$. If $e_{n}(A) \rightarrow T \in \mathcal{L}(X)$ strongly, then $T=0$.

Proof. We have $e_{n}=f_{n}+c_{n} /(1+z)$ for some $f_{n} \in H_{0}^{\infty}\left(S_{\varphi}\right)$ and $c_{n} \in \mathbb{C}$. Since $c_{n}:=\lim _{z \rightarrow 0} e_{n}(z)$ and the convergence is uniform, $c_{n} \rightarrow 0$ and $\lim _{n} f_{n}(A)=T$. Fix $n \in \mathbb{N}$. Since $f_{n} \in H_{0}^{\infty}\left(S_{\varphi}\right)$ there is $\alpha>0$ such that still $h:=f_{n}(z) z^{-\alpha} \in H_{0}^{\infty}\left(S_{\varphi}\right)$. Hence $f_{n}(A)=A^{\alpha} h(A)$, whence $\mathcal{R}\left(f_{n}(A)\right) \subset \mathcal{R}\left(A^{\alpha}\right) \subset \overline{\mathcal{R}(A)}$ (see [17] or [11]). Consequently, $\mathcal{R}(T) \subset \overline{\mathcal{R}(A)}$.

Now we show that also $\mathcal{R}(T) \subset \mathcal{N}(A)$. Let $x=A y \in \mathcal{R}(A)$. Then

$$
(1+A)^{-2} T x=\lim _{n}(1+A)^{-2} f_{n}(A) A y=\lim _{n} \frac{1}{2 \pi \mathrm{i}} \int_{\Gamma} \frac{z}{(1+z)^{2}} f_{n}(z) R(z, A) y \mathrm{~d} z=0 .
$$

This implies $T x=0$. If $x \in \mathcal{D}(A)$ we have $f_{n}(A) x \in \mathcal{D}(A)$ with $A f_{n}(A) x=f_{n}(A) A x$. Since $A$ is closed, $T x \in \mathcal{D}(A)$ with $A T x=T A x=0$. Hence $T \mathcal{D}(A) \subset \mathcal{N}(A)$. Because $X=\mathcal{D}(A)+\mathcal{R}(A)$, we conclude $\mathcal{R}(T) \subset \mathcal{N}(A)$.

In summary, we arrive at $\mathcal{R}(T) \subset \overline{\mathcal{R}(A)} \cap \mathcal{N}(A)$. However, $\overline{\mathcal{R}(A)} \cap \mathcal{N}(A)=0$ holds for every sectorial operator (cf. [11, Chapter I]).

The last result shows that Axiom (Top) is satisfied when we use the restricted calculus $\mathcal{E}_{\text {res }}$ with $\mathcal{H}:=H^{\infty}\left(S_{\varphi}\right)$ and the topology of uniform convergence on $S_{\varphi}$. If $A$ is injective, all of $H^{\infty}\left(S_{\varphi}\right)$ is already regularized by $H_{0}^{\infty}\left(S_{\varphi}\right)$, so we do not gain anything new by the 
topological extension. If $A$ is not injective, we recover all of $\mathcal{E}$, i.e. the original calculus. (For $f \in \mathcal{E}_{0}\left(S_{\varphi}\right)$ consider the approximants $f_{n}(z)=(n z /(1+n z)) f(z)$.) However, the following example shows even more.

Example 5.2. Assume $\mu \in \boldsymbol{M}(0, \infty)$ is a finite (complex) Borel measure on $(0, \infty)$ and consider the function

$$
f(z):=\mu^{\diamond}(z):=\int_{(0, \infty)} \frac{t}{t+z} \mu(\mathrm{d} t) .
$$

It is easy to see that $f \in H^{\infty}\left(S_{\varphi}\right)$ for each $0<\varphi<\pi$. Within the so-called Hirsch calculus (see $[\mathbf{1 7}$, Chapter 4]) one defines

$$
f(A):=\int_{(0, \infty)} t(t+A)^{-1} \mu(\mathrm{d} t)
$$

which certainly is a reasonable definition. When $A$ is injective one can easily show that this is in perfect agreement with our functional calculus. If $A$ is not injective, $\mu^{\diamond} \notin$ $\mathcal{M}\left(S_{\varphi}\right)_{A}$ in general. Take, for example, a sequence $\left(a_{n}\right)_{n \geqslant 2}$ with

$$
a_{n} \geqslant 0, \quad a:=\sum_{n \geqslant 2} a_{n}<\infty, \quad \sum_{n \geqslant 2} a_{n} \log (n+1)=\infty .
$$

and define $\mu:=\sum_{n \geqslant 2} a_{n} \delta_{1 / n}$. Then $\mu$ is a positive measure with total mass $a$. For $f$ defined by (5.3) we have

$$
\begin{aligned}
\int_{0}^{1}|f(t)-f(0)| \frac{\mathrm{d} t}{t} & =\int_{0}^{1}\left|\sum_{n \geqslant 2} a_{n} \frac{-t}{1 / n+t}\right| \frac{\mathrm{d} t}{t}=\sum_{n \geqslant 2} \int_{0}^{1} \frac{a_{n}}{1 / n+t} \mathrm{~d} t \\
& =\sum_{n \geqslant 2} a_{n}\left(\log \left(\frac{1}{n}+1\right)-\log \frac{1}{n}\right) \\
& =\sum_{n \geqslant 2} a_{n} \log (n+1)=\infty .
\end{aligned}
$$

Hence the function $\mu^{\diamond}$ is not contained in $\mathcal{E}\left[S_{\omega}\right]$, i.e. $f(A)$ is not defined by our functional calculus in the case when $A$ is not injective. However,

$$
\mu^{\diamond}(z)=\lim _{n \rightarrow \infty} f_{n}(z) \quad \text { with } f_{n}(z):=\int_{(1 / n, n)} \frac{t}{t+z} \mu(\mathrm{d} t)
$$

and this convergence is uniform on every sector $S_{\varphi}(0<\varphi<\pi)$. The functions $f_{n}$ are contained in $\mathcal{E}_{\text {res }}\left(S_{\varphi}\right)$ and

$$
f_{n}(A)=\int_{(1 / n, n)} t(t+A)^{-1} \mu(\mathrm{d} t)
$$

as is easily proved. Thus the Hirsch calculus can be covered by our AFC

$$
\left.\left(\widetilde{\mathcal{E}_{\text {res }}\left(S_{\varphi}\right.}\right), \mathcal{M}\left(S_{\varphi}\right), \Phi\right)
$$


Unfortunately, topological extensions have considerable drawbacks. A spectral mapping theorem as it is proved in [12] seems to be out of reach if one uses the topologically extended calculus. Also it seems that a nice composition rule (which is the topic of $\S 7$ ) will fail in that case.

\section{Adjoints}

In this section we examine the relation between the functional calculi for $A$ and its adjoint $A^{\prime}$. We begin with an abstract consideration.

Let $(\mathcal{E}, \mathcal{F}, \bullet)$ be a proper abstract functional calculus over the Banach space $X$. Then by $e_{*}:=\left(e_{\bullet}\right)^{\prime}$ a homomorphism

$$
*:\left(e \mapsto e_{*}\right): \mathcal{E} \longrightarrow \mathcal{L}\left(X^{\prime}\right)
$$

is defined. Hence, $(\mathcal{E}, \mathcal{F}, *)$ is an abstract functional calculus over $X^{\prime}$, the so-called dual (functional) calculus. This AFC is proper if and only if there is $e \in \mathcal{E}$ such that $\overline{\mathcal{R}\left(e_{\bullet}\right)}=X$. It is now natural to ask, for which $f \in \mathcal{F}$ the identity $f_{*}=\left(f_{\bullet}\right)^{\prime}$ holds. We obtain the following result.

Proposition 6.1. Let $(\mathcal{E}, \mathcal{F}, \bullet)$ be an $A F C$ with dual calculus $(\mathcal{E}, \mathcal{F}, *)$ and let $f \in \mathcal{F}$. Suppose there is a $\bullet$-regularizer $e \in \mathcal{E}$ for $f$ with the following property.

There exists $\left(f_{n}\right)_{n} \subset \mathcal{F}_{b}$ such that

(i) $f_{n} \bullet \rightarrow I$ strongly on $X$,

(ii) $\mathcal{R}\left(f_{n \bullet}\right) \subset \mathcal{R}\left(e_{\bullet}\right)$.

Then $\mathcal{R}\left(e_{\bullet}\right)$ is dense in $X$, is a core for $f_{\bullet}$ and $f_{*}=\left(f_{\bullet}\right)^{\prime}$.

Proof. It is immediately clear from (i) and (ii) that $\mathcal{R}\left(e_{\bullet}\right)$ is dense in $X$. Hence, $e$ is a regularizer for $f$ in the dual calculus. Let $x \in \mathcal{D}\left(f_{\bullet}\right)$ and $y:=f_{\bullet} x$. Then $x_{n}:=f_{n \bullet} x \rightarrow x$ and $x_{n} \in \mathcal{R}\left(e_{\bullet}\right) \subset \mathcal{D}\left(f_{\bullet}\right)$. Moreover, $f_{\bullet} x_{n}=f_{\bullet} f_{n} \bullet x=f_{n} \bullet y \rightarrow y$. Since $x \in \mathcal{D}\left(f_{\bullet}\right)$ was arbitrary, we see that $\mathcal{R}\left(e_{\bullet}\right)$ is a core for $f_{\bullet}$. This means that $\overline{(e f)_{\bullet} e_{\bullet}^{-1}}=f_{\bullet}$. Then

$$
\begin{aligned}
f_{*} & =\left(e_{*}\right)^{-1}(e f)_{*}=\left[\left(e_{\bullet}\right)^{\prime}\right]^{-1}\left[(e f)_{\bullet}\right]^{\prime}=\left[e_{\bullet}^{-1}\right]^{\prime}\left[(e f)_{\bullet}\right]^{\prime}=\left[(e f)_{\bullet} e_{\bullet}^{-1}\right]^{\prime} \\
& =\left[\overline{(e f)_{\bullet} e_{\bullet}^{-1}}\right]^{\prime}=(f \bullet)^{\prime},
\end{aligned}
$$

where we have used some elementary rules for computations with adjoints (see [11, Appendix A]).

Let us return to sectorial operators. Suppose $A \in \operatorname{Sect}(\omega)$ and $\overline{\mathcal{D}(A)}=X$. This extra condition ensures that $A^{\prime}$ is again single valued, whence also $A^{\prime} \in \operatorname{Sect}(\omega)$.

Lemma 6.2. Let $A \in \operatorname{Sect}(\omega)$ with $\overline{\mathcal{D}(A)}=X$. Then the PFC for $A^{\prime}$ is the dual of the PFC for $A$, i.e. $f\left(A^{\prime}\right)=f(A)^{\prime}$ for every $f \in \mathcal{E}\left[S_{\omega}\right]$. 
Proof. Since $\left[(1+A)^{-1}\right]^{\prime}=\left(1+A^{\prime}\right)^{-1}$ we can assume $f \in \mathcal{E}_{0}\left[S_{\omega}\right]$ without loss of generality. Hence

$$
f(A)=\frac{1}{2 \pi \mathrm{i}} \int_{\Gamma} f(z) R(z, A) \mathrm{d} z
$$

for a certain $\Gamma$. Thus

$$
\begin{aligned}
f(A)^{\prime} & =\left(\frac{1}{2 \pi \mathrm{i}} \int_{\Gamma} f(z) R(z, A) \mathrm{d} z\right)^{\prime}=\frac{1}{2 \pi \mathrm{i}} \int_{\Gamma} f(z) R(z, A)^{\prime} \mathrm{d} z \\
& =\frac{1}{2 \pi \mathrm{i}} \int_{\Gamma} f(z) R\left(z, A^{\prime}\right) \mathrm{d} z=f\left(A^{\prime}\right) .
\end{aligned}
$$

Combining this result with Proposition 6.1 we can prove the identity $f(A)^{\prime}=f\left(A^{\prime}\right)$ for certain classes of functions $f$.

Proposition 6.3. Let $A \in \operatorname{Sect}(\omega)$ with $\overline{\mathcal{D}(A)}=X, \omega<\varphi<\pi$ and $f \in \mathcal{O}\left(S_{\varphi}\right)$. Assume that $f$ satisfies the conditions (i) and (ii) of Proposition 4.5. Then $f(A)^{\prime}=f(A)^{\prime}$.

The same conclusion holds if $\overline{\mathcal{R}(A)}=X$ and both $f, f(1 / z)$ satisfy the condition (ii) of Proposition 4.5.

Proof. In the first case one can pick a regularizer $e$ of the form $e(z):=\mathrm{e}^{-s z^{\alpha}}$ with $s>0, \alpha>0$ and $\alpha \varphi<\pi / 2$. Choose $\alpha<\beta<\pi / 2 \varphi$ and define $f_{n}(z):=\mathrm{e}^{-(1 / n) z^{\beta}}$. Then $B:=A^{\beta}$ is still densely defined, whence $f_{n}(A)=\mathrm{e}^{-(1 / n) B} \rightarrow I$ strongly (see $[\mathbf{1 5}]$ or [11]). Moreover, $\mathrm{e}^{s z^{\alpha}-(1 / n) z^{\beta}} \in \mathcal{E}\left(S_{\varphi}\right)$, whence $\mathcal{R}(e(A)) \supset \mathcal{R}\left(f_{n}(A)\right)$ for all $n$. Hence Proposition 6.1 is applicable. The second assertion is proved similarly.

\section{The composition rule}

In all that follows, we use only the $\operatorname{AFC}\left(\mathcal{E}_{\text {res }}\left[S_{\omega}\right], \mathcal{M}\left[S_{\omega}\right], \Phi\right)$, not the original calculus and not its topological extension. We will study the so-called composition rule by which we mean an identity of the form

$$
(f \circ g)(A)=f(g(A))
$$

It is this identity which we consider the most fundamental, making the functional calculus 'work'. As a matter of fact, the rule as it stands does not make sense, unless we require some additional hypotheses. Basically, we make the following requirements:

(i) $g$ maps a sector into another sector;

(ii) $A$ is sectorial;

(iii) $g(A)$ is defined by the restricted calculus and is also sectorial.

More precisely, we obtain the following result. 
Theorem 7.1. Let $0 \leqslant \omega<\varphi<\pi, A \in \operatorname{Sect}(\omega)$ and $g \in \mathcal{M}\left(S_{\varphi}\right)_{\text {res, } A}$. Assume that $g(A) \in \operatorname{Sect}\left(\omega^{\prime}\right)$ with $g\left(S_{\varphi}\right) \subset \overline{S_{\omega^{\prime}}} \cup\{\infty\}$. Take $f \in \mathcal{M}\left[S_{\omega^{\prime}}\right]_{\text {res }, g(A)}$. Then $f \circ g \in \mathcal{M}\left(S_{\varphi}\right)_{A}$ and the composition rule

$$
(f \circ g)(A)=f(g(A))
$$

holds.

Note that by the open mapping theorem, if $g$ has the required mapping property (namely, $g\left(S_{\varphi}\right) \subset \overline{S_{\omega^{\prime}}} \cup\{\infty\}$ ), $g$ cannot have zeros or poles within $S_{\varphi}$, unless $g=0$ is constant. Hence if $g \neq 0$, then $g$ and $g^{-1}$ are both holomorphic on $S_{\varphi}$ and $g\left(S_{\varphi}\right) \subset S_{\psi}$ for every $\omega<\psi<\pi$.

Lemma 7.2. Let $\omega, \varphi, A$ and $g \neq 0$ be as above. If $f \in \mathcal{M}\left[S_{\omega^{\prime}}\right]$, then $f \circ g \in \mathcal{M}\left(S_{\varphi}\right)$. If $f \in \mathcal{E}_{\text {res }}\left[S_{\omega^{\prime}}\right]$, then $f \circ g \in H^{\infty}\left(S_{\varphi}\right)_{\mathrm{res}, A}$.

Proof. The first statement is clear by the remarks preceding this lemma. Obviously, also $f \circ g \in H^{\infty}\left(S_{\varphi}\right)$ holds for every $f \in \mathcal{E}\left[S_{\omega^{\prime}}\right]$.

If $A$ is injective, there is nothing more to show. Suppose $A$ is not injective. Then by Lemma 4.2 the function $g$ must have a limit at 0 with $g(z)-g(0)=O\left(|z|^{\alpha}\right)$ for some $\alpha>0$ as $z \rightarrow 0$. Since $f \in \mathcal{E}_{\text {res }}$, the function $f \circ g$ will have a similar limit behaviour at 0 . Hence $f \circ g$ is regularizable by $(1+z)^{-1}$, and the lemma is proved.

Lemma 7.3. Let $\omega, \varphi, A, g$ be as above. Suppose the composition rule $f(g(A))=$ $(f \circ g)(A)$ is true for every $f \in \mathcal{E}_{\text {res }}\left[S_{\omega^{\prime}}\right]$. Then it is true for every $f \in \mathcal{M}\left[S_{\omega^{\prime}}\right]_{\mathrm{res}, g(A)}$.

Proof. Let $f \in \mathcal{M}\left[S_{\omega^{\prime}}\right]_{\text {res }, g(A)}$ and let $e \in \mathcal{E}_{\text {res }}$ be a regularizer for $f$ with respect to $g(A)$. From Lemma 7.2 it follows that $f \circ g \in \mathcal{M}\left(S_{\varphi}\right)$. Lemma 7.2 together with the hypothesis implies that $(e \circ g)(A)=e(g(A))$ is bounded and injective. Moreover, $(f \circ g)(e \circ g)=(e f) \circ g$ and $[(e f) \circ g](A)=(e f)(g(A)) \in \mathcal{L}(X)$. Now an appeal to Proposition 3.7 shows that $f \circ g \in \mathcal{M}\left(S_{\varphi}\right)_{\text {res }, A}$. Moreover,

$$
\begin{aligned}
(f \circ g)(A) & =(e \circ g)(A)^{-1}[(e \circ g)(f \circ g)](A)=e(g(A))^{-1}[(e f) \circ g](A) \\
& =e(g(A))^{-1}(e f)(g(A))=f(g(A)) .
\end{aligned}
$$

We take the final step.

Lemma 7.4. Let $\omega, \varphi, A, g$ be as above. Then the composition rule $f(g(A))=$ $(f \circ g)(A)$ is true for every $f \in \mathcal{E}_{\text {res }}\left[S_{\omega^{\prime}}\right]$.

Proof. Let $f \in \mathcal{E}_{\text {res }}\left[S_{\omega^{\prime}}\right]$. Then there is a constant $c \in \mathbb{C}$ and $f_{1} \in H_{0}^{\infty}\left[S_{\omega^{\prime}}\right]$ such that $f=c /(1+z)+f_{1}$. If $f=c /(1+z)$, then the assertion is contained in (vi) of Theorem 3.6. Hence we can assume without restriction that $f \in H_{0}^{\infty}$. For $\lambda \notin \overline{S_{\omega^{\prime}}}$ the function $(\lambda-g(z))^{-1}$ certainly is bounded and holomorphic on $S_{\varphi}$. Now

$$
f(g(A))=\frac{1}{2 \pi \mathrm{i}} \int_{\Gamma^{\prime}} f(\lambda) R(\lambda, g(A)) \mathrm{d} \lambda=\frac{1}{2 \pi \mathrm{i}} \int_{\Gamma^{\prime}} f(\lambda)\left(\frac{1}{\lambda-g(z)}\right)(A) \mathrm{d} \lambda,
$$

where $\Gamma^{\prime}$ surrounds the sector $\overline{S_{\omega^{\prime}}}$. We consider two cases: 
(i) $A$ is injective;

(ii) $A$ is not injective.

The first case is easier to handle. If $A$ is injective, we can use $\tau(z):=z(1+z)^{-2}$ as a regularizer, let $\Gamma$ surround $S_{\omega}$ within $S_{\varphi}$ and compute

$$
\begin{aligned}
f(g(A)) & =\tau(A)^{-1} \tau(A) f(g(A))=\tau(A)^{-1} \frac{1}{2 \pi \mathrm{i}} \int_{\Gamma^{\prime}} f(\lambda) \tau(A) R(\lambda, g(A)) \mathrm{d} \lambda \\
& =\tau(A)^{-1} \frac{1}{2 \pi \mathrm{i}} \int_{\Gamma^{\prime}} f(\lambda)\left(\frac{z}{(1+z)^{2}(\lambda-g(z))}\right)(A) \mathrm{d} \lambda \\
& =\tau(A)^{-1} \frac{1}{(2 \pi \mathrm{i})^{2}} \int_{\Gamma^{\prime}} \int_{\Gamma} f(\lambda) \frac{z}{(1+z)^{2}(\lambda-g(z))} R(z, A) \mathrm{d} z \mathrm{~d} \lambda \\
& \stackrel{(1)}{=} \tau(A)^{-1} \frac{1}{2 \pi \mathrm{i}} \int_{\Gamma} \frac{f(g(z)) z}{(1+z)^{2}} R(z, A) \mathrm{d} z \\
& =\tau(A)^{-1}(f(g(z)) \tau(z))(A)=(f \circ g)(A) .
\end{aligned}
$$

Equality (1) is an application of Cauchy's integral theorem. Before, one has to interchange the order of integration. To justify this, note that the function

$$
\frac{f(\lambda)}{(\lambda-g(z))(1+z)^{2}}=\frac{\lambda}{\lambda-g(z)} \frac{f(\lambda)}{\lambda} \frac{1}{(1+z)^{2}}
$$

is product integrable on $\Gamma \times \Gamma^{\prime}$ since the first factor is uniformly bounded.

To cover the second case, assume that $A$ is not injective. Then by Lemma $4.2, g$ must have a finite limit $c:=g(0)$ at 0 which is approached at least polynomially. Define $g_{1}(z):=g(z)-g(0)$ and choose a regularizer $e \in \mathcal{E}$ for $g_{1}$. Then $e g_{1} \in H_{0}^{\infty}$. Furthermore, for $\lambda \notin \overline{S_{\omega^{\prime}}}$ the bounded function $\left[(\lambda-g(z))^{-1}-(\lambda-c)^{-1}\right]$ is also regularized by $e$. Thus we compute

$$
\begin{aligned}
f(g(A))= & e(A)^{-1} e(A) f(g(A))=e(A)^{-1} \frac{1}{2 \pi \mathrm{i}} \int_{\Gamma^{\prime}} f(\lambda) e(A) R(\lambda, g(A)) \mathrm{d} \lambda \\
= & e(A)^{-1} \frac{1}{2 \pi \mathrm{i}} \int_{\Gamma^{\prime}} f(\lambda)\left(\frac{e(z)}{\lambda-g(z)}\right)(A) \mathrm{d} \lambda \\
= & e(A)^{-1} \frac{1}{2 \pi \mathrm{i}} \int_{\Gamma^{\prime}} f(\lambda)\left(\frac{g_{1}(z) e(z)}{(\lambda-g(z))(\lambda-g(0))}+\frac{e(z)}{\lambda-c}\right)(A) \mathrm{d} \lambda \\
= & e(A)^{-1} \frac{1}{2 \pi \mathrm{i}} \int_{\Gamma^{\prime}} f(\lambda)\left(\frac{\left(g_{1} e\right)(z)}{(\lambda-g(z))(\lambda-c)}\right)(A)+\frac{f(\lambda)}{\lambda-c} e(A) \mathrm{d} \lambda \\
= & e(A)^{-1} \frac{1}{2 \pi \mathrm{i}} \int_{\Gamma^{\prime}} f(\lambda)\left(\frac{\left(g_{1} e\right)(z)}{(\lambda-g(z))(\lambda-c)}\right)(A) \mathrm{d} \lambda \\
& +e(A)^{-1} \frac{1}{2 \pi \mathrm{i}} \int_{\Gamma^{\prime}} \frac{f(\lambda)}{\lambda-c} \mathrm{~d} \lambda e(A) .
\end{aligned}
$$


The second summand equals $f(c)$ by Cauchy's theorem. The first satisfies

$$
\begin{aligned}
e(A)^{-1} & \frac{1}{2 \pi \mathrm{i}} \int_{\Gamma^{\prime}} f(\lambda)\left(\frac{\left(g_{1} e\right)(z)}{(\lambda-c)(\lambda-g(z))}\right)(A) \mathrm{d} \lambda \\
& =e(A)^{-1} \frac{1}{2 \pi \mathrm{i}} \int_{\Gamma^{\prime}} f(\lambda) \frac{1}{2 \pi \mathrm{i}} \int_{\Gamma} \frac{\left(g_{1} e\right)(z)}{(\lambda-c)(\lambda-g(z))} R(z, A) \mathrm{d} z \mathrm{~d} \lambda \\
& \stackrel{(1)}{=} e(A)^{-1} \frac{1}{2 \pi \mathrm{i}} \int_{\Gamma}\left(\frac{1}{2 \pi \mathrm{i}} \int_{\Gamma^{\prime}} f(\lambda)\left[\frac{1}{\lambda-g(z)}-\frac{1}{\lambda-c}\right] \mathrm{d} \lambda\right) e(z) R(z, A) \mathrm{d} z \\
& \stackrel{(2)}{=} e(A)^{-1} \frac{1}{2 \pi \mathrm{i}} \int_{\Gamma}(f(g(z))-f(c)) e(z) R(z, A) \mathrm{d} z \\
& \stackrel{(3)}{=} e(A)^{-1}[(f(g(z))-f(c)) e(z)](A) \\
& =e(A)^{-1}((f \circ g)(A)-f(c)) e(A)=(f \circ g)(A)-f(c),
\end{aligned}
$$

where we used Fubini's theorem in (1) and Cauchy's theorem in (2). To justify the application of Fubini's theorem in (1) one has, after estimating the resolvent, to consider the function

$$
F(\lambda, z):=\frac{f(\lambda)\left(g_{1} e\right)(z)}{(\lambda-c)(\lambda-g(z)) z}
$$

and prove its product integrability. The representation

$$
F(\lambda, z)=\left(\frac{f(\lambda)}{\lambda}\right)\left(\frac{\lambda}{(\lambda-c)(\lambda-g(z))}\right)\left(\frac{\left(g_{1} e\right)(z)}{z}\right)
$$

shows that $c \neq 0$ is harmless: $\lambda /(\lambda-g(z))$ is uniformly bounded because of the conditions $g(z) \in \overline{S_{\omega^{\prime}}}$ and $\lambda \in \Gamma^{\prime}$. (Recall that $\left(e g_{1}\right) \in H_{0}^{\infty}$.) If $c=0$ (hence $g_{1}=g$ ), we write

$$
\begin{aligned}
F(\lambda, z) & =\left(\frac{f(\lambda)}{\lambda}\right)\left(\frac{1}{\lambda-g(z)}\right)\left(\frac{(g e)(z)}{z}\right) \\
& =\left(\frac{f(\lambda)}{\lambda^{1+\alpha}}\right)\left(\frac{\lambda^{\alpha} g(z)^{1-\alpha}}{\lambda-g(z)}\right)\left(\frac{g(z)^{\alpha} e(z)}{z}\right) .
\end{aligned}
$$

(Recall that $g$ has no poles in $S_{\varphi}$.) Here, $0<\alpha<1$ is chosen in such a way that the first factor remains integrable. Then, the middle term is still uniformly bounded. It is easily seen that also $e g^{\alpha} \in H_{0}^{\infty}$, whence $F$ is integrable.

Corollary 7.5. Let $A \in \operatorname{Sect}(\omega)$ be injective and $f \in \mathcal{M}\left[S_{\omega}\right]$. Then

$$
f \in \mathcal{M}\left[S_{\omega}\right]_{A^{-1}} \quad \Longleftrightarrow f\left(z^{-1}\right) \in \mathcal{M}\left[S_{\omega}\right]_{A}
$$

and in this case $f\left(A^{-1}\right)=f\left(z^{-1}\right)(A)$.

Proof. Note that, since both $A$ and $A^{-1}$ are injective, restricted and original calculus are the same. Assume $f \in \mathcal{M}\left[S_{\omega}\right]_{A^{-1}}$ and let $\psi>\omega$ such that $f \in \mathcal{M}\left(S_{\psi}\right)$. Choose $\omega<$ $\varphi<\psi$ and set $\omega^{\prime}:=\varphi$. Clearly, $A^{-1} \in \operatorname{Sect}\left(\omega^{\prime}\right), g(z):=z^{-1} \in \mathcal{M}\left(S_{\varphi}\right)_{A}$ and $g\left(S_{\varphi}\right) \subset S_{\omega^{\prime}}$. Applying Theorem 7.1 yields $f\left(z^{-1}\right) \in \mathcal{M}\left(S_{\varphi}\right)_{A}$ and $f\left(z^{-1}\right)(A)=f\left(A^{-1}\right)$. Interchanging the roles of $A$ and $A^{-1}$ proves correct the other implication. 
Example 7.6. Let us illustrate the use of the composition rule. By elementary calculations one sees that for $A \in \operatorname{Sect}(\omega)$ and $\varepsilon>0$ also $A+\varepsilon, A(A+\varepsilon)^{-1} \in \operatorname{Sect}(\omega)$. The composition rule and (iii) of Theorem 3.6 yield

$$
\left[(A+\varepsilon)^{-1}\right]^{\alpha}=\left[(A+\varepsilon)^{\alpha}\right]^{-1}=(A+\varepsilon)^{-\alpha} \text { and }\left[A(A+\varepsilon)^{-1}\right]^{\alpha}=A^{\alpha}(A+\varepsilon)^{-\alpha}
$$

for $\operatorname{Re} \alpha>0$. This shows readily $\mathcal{D}\left((A+\varepsilon)^{\alpha}\right) \subset \mathcal{D}\left(A^{\alpha}\right)$. (The other inclusion is also easy, see $[\mathbf{1 1}]$.) Another instance of the composition rule is

$$
\mathrm{e}^{-t z^{\alpha}}(A)=\mathrm{e}^{-t z}\left(A^{\alpha}\right)=\mathrm{e}^{-t A^{\alpha}}
$$

where $0<\alpha<\pi / 2 \omega$. Here one uses the fact that $A^{\alpha} \in \operatorname{Sect}(\alpha \omega)$ for $0<\alpha<\pi / \omega$, an easy functional calculus proof of which can be found in [11]. Taking this for granted, the power law

$$
\left(A^{\alpha}\right)^{\beta}=A^{\alpha \beta} \quad(0<\alpha<\pi / \omega, \operatorname{Re} \beta>0)
$$

is also just another application of the composition rule. If $A$ is injective, one can define $\log A:=(\log z)(A)$ and obtains the identities

$$
\log \left(A^{-1}\right)=-\log A \quad \text { and } \quad \log \left(A^{\alpha}\right)=\alpha \log A \quad(0<\alpha<\pi / \omega) .
$$

Remark 7.7. Actually, a slightly more general version of the composition rule is true. Namely, if $A$ is injective, we can admit $f \in \mathcal{M}_{g(A)}$ and do not have to restrict to $f$ 's from $\mathcal{M}_{\text {res, } A}$. This is clear by browsing through the proofs. Actually, this remark would be superfluous if we could guarantee that $g(A)$ is injective if $A$ is. However, we do not know if this is true (cf. [12, Corollary 6.6]).

\section{Concluding remarks}

The considerations of this paper show paradigmatically how (holomorphic) functional calculi can be constructed, which results about them can be expected (like the composition rule) and how they can be proved. The author has carried out such a construction for so-called strip type operators, to cover logarithms of injective sectorial operators (see $[\mathbf{9}],[\mathbf{1 0}]$ or $[\mathbf{1 1}]$ ). Also, 'half-plane-type' operators, with the spectrum contained in a half-plane and the resolvent bounded on the complement, seem to be interesting (semigroup generators!) and of course a functional calculus can be constructed along the lines given here. In fact, the whole setting of [5] and the calculus for 'almost sectorial operators' constructed in $[\mathbf{1 8}]$ also fall under this scheme.

However, the Phillips calculus for semigroup generators and other 'real' constructions as in $[\mathbf{8}]$ or $[\mathbf{1 9}]$ are not covered. In fact, a general theory of functional calculus, whatever this means precisely, covering and connecting nicely all known sorts of calculi seems to be far away. All we can achieve up to now is to construct functional calculi for special classes of operators, then prove coherence and composition rules for each pair of them. 


\section{References}

1. D. Albrecht, X. Duong And A. McIntosh, Operator theory and harmonic analysis, in Instructional Workshop on Analysis and Geometry, Part III, Canberra, 1995, pp. 77-136 (Australian National University, Canberra, 1996).

2. W. AREndt, Semigroups and evolution equations: functional calculus, regularity and kernel estimates, in Handbook of differential equations (ed. C. M. Dafermos and E. Feireisl), pp. 1-85 (Elsevier/North-Holland, 2004).

3. W. G. BADE, An operational calculus for operators with spectrum in a strip, Pac. J. Math. 3 (1953), 257-290.

4. M. Cowling, I. Doust, A. McIntosh And A. Yagi, Banach space operators with a bounded $H^{\infty}$ functional calculus, J. Austral. Math. Soc. A 60(1) (1996), 51-89.

5. R. DELAUBenfels, Unbounded holomorphic functional calculus and abstract Cauchy problems for operators with polynomially bounded resolvents, J. Funct. Analysis 114 (1993), 348-394.

6. R. Denk, M. Hieber AND J. Prüss, R-boundedness, Fourier multipliers and problems of elliptic and parabolic type, Memoirs of the American Mathematical Society, vol. 166 (American Mathematical Society, Providence, RI, 2003).

7. N. Dunford And J. T. Schwartz, Linear operators, I, General theory, Pure and Applied Mathematics, vol. 6 (Interscience, 1958).

8. J. E. GALÉ AND T. PYTLIK, Functional calculus for infinitesimal generators of holomorphic semigroups, J. Funct. Analysis 150 (1997), 307-355.

9. M. HAASE, A characterization of group generators on Hilbert spaces and the $H^{\infty}$-calculus, Semigroup Forum 66 (2003), 288-304.

10. M. HAASE, Spectral properties of operator logarithms, Math. Z. 245 (2003), 76-779.

11. M. HAASE, The functional calculus for sectorial operators, to appear in Operator theory: advances and applications (Birkhäuser, Basel, 2005) (preliminary version available at http://www.mathematik.uni-ulm.de/m5/haase.)

12. M. HAASE, Spectral mapping theorems for holomorphic functional calculi, J. Lond. Math. Soc., to appear.

13. N. J. Kalton And L. Weis, The $H^{\infty}$-calculus and sums of closed operators, Math. Ann. 321 (2001), 319-345.

14. P. C. Kunstmann And L. Weis, Maximal $L_{p}$-regularity for parabolic equations, Fourier multiplier theorems and $H^{\infty}$-functional calculus, in Functional Analytic Methods for Evolution Equations, Levico Terme, 2001, Lecture Notes in Mathematics, vol. 1855, pp. 65-312 (Springer, 2004).

15. A. LunARDi, Analytic semigroups and optimal regularity in parabolic problems, Progress in Nonlinear Differential Equations and their Applications, vol. 16 (Birkhäuser, Basel, 1995).

16. A. McIntosh, Operators which have an $H_{\infty}$ functional calculus, in Miniconference on Operator Theory and Partial Differential Equations, North Ryde, 1986, pp. 210-231 (Australian National University, Canberra, 1986).

17. C. Martínez Carracedo And M. Sanz Alix, The theory of fractional powers of operators (North-Holland, 2001).

18. F. Periago And B. Straub, A functional calculus for almost sectorial operators and applications to abstract evolution equations, J. Evol. Eqns 2(1) (2002), 41-68.

19. M. Uiterdijk, A functional calculus for analytic generators of $C_{0}$-groups, Integ. Eqns Operat. Theory 36 (2000), 349-369. 